\title{
Kleiner Beitrag zur atavistischen Regeneration der Scheren beim Flußkrebse.
}

\author{
Von \\ Józef Nusbaum \\ in Lemberg. \\ (Zoologisches Institut der Universität.) \\ Mit 2 Figuren im Text. \\ Eingegangen am 21. März 1907.
}

Dr. Eugen Schultzi) hat im vorigen Jahre eine interessante Untersuchung über die atavistische Regeneration der Scheren beim Flußkrebse, Steinkrebse und andern Arten des Astacus veröffentlieht. Er bestätigt die ältere Beobachtung von Kessler, daß beim Astacus pachypus besonders beim männlichen Individuum eine der vorderen Scheren sehr oft viel kleiner als die andre entwickelt ist, und daß diese kleinere Schere fast immer der Form nach von der größeren sich unterscheidet, und der Schere von Astacus leptodactylus sehr ähnlich erscheint. KessLer nahm richtig an, daß die kleinere Schere eine regenerierte Schere darstellt, und da nach KessLen der Astacus leptodactylus phylogenetiseh eine Urform andrer russischer Krebsarten und somit auch des A. pachypus bildet, so sieht er in der Art und Weise der Scherenregeneration eine Tendenz, zur früheren Form zurückzukehren (Atavismus). SchuLtz erwähnt dann eine Beobachtung von E. K. roN BAER, der beim A. pachypus gleicherweise eine regenerierte Schere nach dem Typus von $A$. leptodactylus beobachtete, und dann die Beobachtung von Skonmow, der kleine Scheren nicht nur bei A. pachypus, sondern auch beim $A$. fluviatilis, A. colchicus und A. Kesslevi nach dem Typus der Schere von $A$. leptodactylus gebaut gesehen hat.

1) E. Schultz, Über atavistische Regeneration bei Flußkrebsen. Archiv f. Entw.-Mech. Bd. XX. 1906. 
SchULTz untersuchte nun näher das reiche Material von SkoRrKow und kam zum interessanten Schlusse, daß bei allen genannten Formen die kleinere, also sehr wahrscheinlich die regenerierte Schere nach dem Typus der Leptodactylus-Schere gebaut ist.

Die Schere von Leptodactylus unterscheidet sich bekanntlich von derjenigen andrer Krebsarten dadurch, daß in derselben die Hand abgeflacht, schmäler und länger ist, Index und Dactylopodit legen sich ziemlich vollkommen aneinander, da die Einbuchtnng am inneren Rande des Index zwischen zwei Höckern fehlt und diese Höcker selbst kaum entwickelt sind. Die Finger sind lang.

In der Art Astacus leptodactylus unterscheidet KESsLER eine Varietät colchica, welche er eben als die Urform aller »russischen" Krebsarten betrachtet, und bei welcher die Hand länger und schmaler als bei der gewöhnlichen Form von $A$. leptodactylus ist und die Finger der Scheren nicht etwas halbmondförmig gebogen, sondern ganz gerade sind. Und nun fand Schultz, daB die kleinere Schere bei 7 Exemplaren von A. leptodactylus nicht nur die typischen Artmerkmale von $A$. leptodactylus trug, sondern am meisten der Schere des A. leptodactylus var. colchica ähnlich war. Es tritt also auch in dieser Hinsicht eine Art atavistischer Regeneration hervor.

Der atavistischen Regeneration habe ich ${ }^{1}$ ) in einigen meiner friheren Arbeiten eine wichtige Bedeutung zugeschrieben. Ich verweise auf meine Schriften über die Regeneration der Würmer und der Fische, wo ich als eine besondere Art der Regeneration »eine atavistische unterschieden und Beispiele einer solchen (z. B. ectodermale Bildung der Muskulatur bei der Regeneration der Würmer, eine zusammenhängende Bildung cines Teiles der Muskulatur und des Bauchnervenstranges bei einigen Polychäten) angeführt habe; es tritt manchmal ein Rückschlag zu phylogenetisch sehr entfernten, aber immer niedrigeren Tiergruppen auf.

Die Anschauungen von Kessler und Schultz scheinen mir sehr interessant und durchaus ganz berechtigt zu sein, und ich will deshalb meinerseits einige diesbezügliche Beobachtungen mitteilen, welche eine weitere Stuitze für die Richtigkeit dieser Anschaungen bilden möchten.

Ich habe nämlich in den Sammlungen des hiesigen Zoologischen Institutes ein Exemplar von Astacus fluviatilis gefunden, bei welchem

1) J. Nusbaum, Vergleichende Regenerationsstud. I, II, III. 1901. 1904. 1905. 
die linke Schere groß, die rechte dagegen sehr klein ist und alle Zeichen einer Schere des Typus von A. leptodactylus besitzt (Fig. 1); die Hand ist hier abgeflacht, schmal, verlängert, am inneren Rande des Index ist keine Spur von der Einbuchtung und von den beiden großen Höckem vorhanden, so daß beide Teile sich vollkommen beim Schlusse der Schere miteinander berühren; die Finger sind schmal und etwas halbmondförmig gebogen.

Fig. 1.

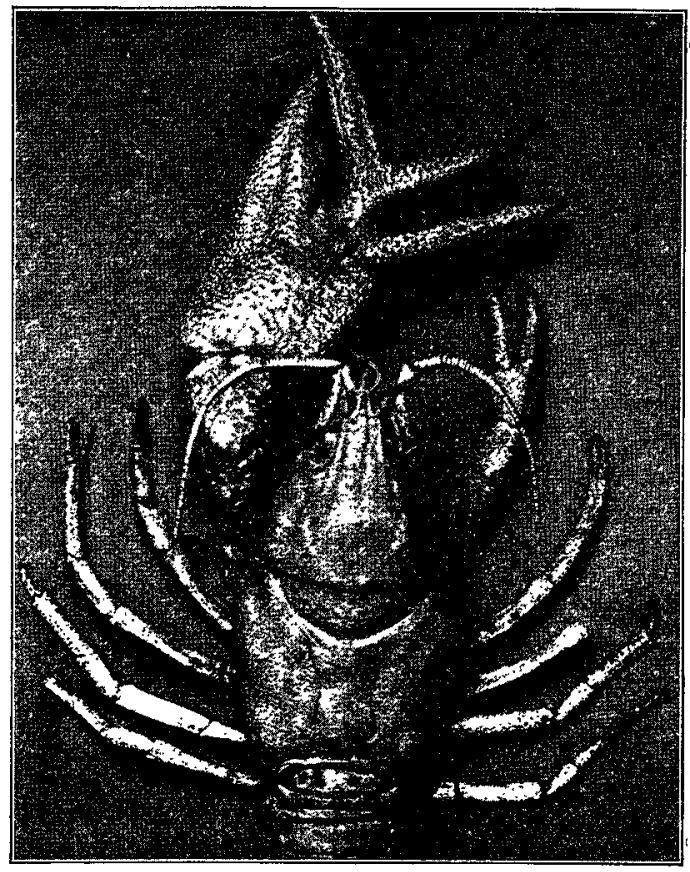

Photographische Aufuahmo eines A. fuviatilis (verkleinert). Die rechte Schere klein, die linke mit einer überzähligen Schere versehen.
Außerdem ist das vorliegende Exemplar noch in andern Hinsichten interessant. Und zwar am Dactylopoditen der linken Schere ist an der Basis derselben, an der inneren (medianen) Seite eine überzählige, unvollkommene Schere entwickelt, ans zwei Fingern bestehend, welche unbeweglich und ohne jede Articulation aus dem Dactylopoditen hervorwächst. Diese überzählige Schere entstand sehr wahrscheinlich gleicherweise infolge einer Wunde, die der Krebs zufällig in der Periode der Häutung, wenn die Chitin-

schicht äußerst dünn und zart war, erhalten hat (vielleicht durch einen Raubfiseh oder eine Raublarve eines Wasserkäfers). In dieser Extraschere ist der äußere, an der Basis etwas verdickte Ast als Index, der innere, etwas diunnere als Dactylopodit zu bezeichnen; und es zeigt sich nun, daß die beiden Finger lang und schmal sind, daß keine Ausbuchtung und keine für $A$. fluviatilis so charakteristischen Höcker am inneren Rande des Index vorhanden sind, und außerdem, daß die Finger keine halbmondförmige Beugung zeigen, sondern gerade verlaufen. Die überzählige, obwohl unvollkommen ausgebildete 
Schere zeigt also alle Charaktere der Finger der Schere von $A$. leptodactylus var. colchica, so daß wir hier wieder eine Bestätigung. der Regel vor uns haben.

Wir muissen beiftugen, daß die mutmaßliche Verwundung der Schere eine noch andre accessorische Bildung hervorgerufen hat, und zwar auf der Unterseite des Index (Fig. 2) der großen Schere an der Grenze des Dactylopoditen existiert hier noch eine Anlage einer zweiten accessorischen Schere in Gestalt eines pyramidenförmigen Höckers, dessen nach unten gerichteter Gipfel in einem inneren, längeren, spitzigen und einem äußeren, stumpfen, kürzeren Zapfen ausläuft.

Bei der Regeneration der Organe finden wir bekanntlich sebr oft eine hypertrophische Bildung des reFig. 2. generierten Gewebes. Diese hypertrophisehe Gewebsbildung kann nun entweder eine $\mathrm{Ver}$ größerung des betreffenden, in der Regeneration begriffenen Organs (eine solche Hypertrophie habe ich z. B. mebrmals bei der Regeneration der Polychäten beobachtet, wo

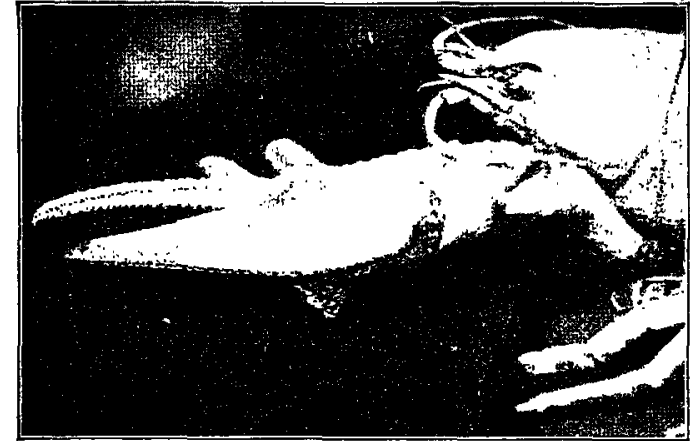

Photographische Aufnahme der linken Schere von demselben FInBkrebse, vom änßeren Rande gesehen (verkleinert). einzelne regenerierte Parapodien viel größer erschienen als normal), oder die Bildung einer größeren Zahl betreffender Organe, als normal, bedingen (z. B. die Bildung zweier Schwänze anstatt eines einzigen bei der Eidechse, die Bildung überzähliger Extremitäten bei den Amphibien). Ich möchte also im allgemeinen: 1) eine qualitative Hypertrophie und 2) eine quantitative Hypertrophie der regenerierten Organe unterscheiden.

In vielen Fällen reicht jedoch das regenerierte Gewebe nicht, um den verloren gegangenen Körperabschnitt oder das verlorene Organ in der vollkommenen Größe zu regenerieren; die regenerierten Teile erscheinen dann kleiner als gewöhnlich. Ich möchte das als Meiotrophie (meion $=$ zu klein') bezeichnen.

Die Regeneration der rechten Schere im oben beschriebenen 
Falle möchten wir also als eine atavistisch-qualitativ-meiotrophische bezeichnen, in der rechten Schere dagegen haben wir eine atavistisch-quantitativ-hypertrophische Regeneration vor uns.

Ich muß noch bemerken, daß überzählige Scheren und Scherenteile schon mehrmals bei verschiedenen Crustaceen beschrieben worden sind. Was den Flußkrebs und andre Arten des Krebses anbelangt, so sind auch in dieser Hinsicht schon mehrere diesbezuigliche Fälle beschrieben worden.

So hat z. B. Lukas (Ann. Soc. entomol. Fr. 1844. Sér. 2) in der linken Schere eines Flußkrebses einen überzähligen kegelförmigen Anhang nach außen von der Basis des Index beschrieben. Dann beschrieb J. KanoLI (Term. Füzetek 1877) in der linken Schere beim Astacus leptodlactylus zwei $»$ Extra-Dactylopoditen $«$, auf dem normalen Dactylopoditen aufsitzend; ich möchte aber diese zwei uiberzähligen Finger eher als dem Index und dem Dactylopoditen der überzähligen Schere entsprechend ansehen.

Sehr interessant ist der ron Canconi beschriebene Fall (Rend. R. Instit. Lombard. 1883. XVI.); in der linken Schere von Astacus fluviatilis entsprang aus dem normalen Carpopoditen ein überzähliger, nach außen gerichteter Carpopodit mit drei Extrascheren. SouberDIN beschrieb (Comptes Rendus. LX. 1865) eine Anomalie in der linken. Schere von Astacus fluviatilis, und zwar aus der Basis des normalen Dactylopoditen und der Hand wachsen hier folgende überzählige Teile hervor: ein rechter und linker Extradactylopodit und ein rechter und linker Extraindex, unvollkommen abgegrenzt voneinander. Es gibt noch einige andre ähnliche Fälle, welche in dem wichtigen Werke Batesons » Materials for the Study of Variation «, 1894, angeführt sind, dem ich im Mangel der betreffenden Literatur auch die oben angeführten Data entnommen habe.

\section{Nachtrag.}

Eingegangen am 11. April 1907.

Der obige Beitrag war schon zum Druck überreicht, als ich die deutsche Ausgabe von T. H. Morgans Werk: "Regeneration" erhalten und die Bemerkungen Moszkowskis über die »atavistischen « Erscheinungen bei der Regeneration der Krebsscheren durchgelesen habe.

Moszkowski ist der Meinung, daß, obwohl es in den von 
Kessler und SchulTz beschriebenen Fällen sich aller Wahrscheinlichkeit nach um regenerierte Scheren handelt, man jedoch hier keineswegs von einer atavistischen Regeneration reden kann. Die Argumentation Moszkowskis charakterisiert der folgende Passus: „Wie sollen wir die Herbstschen Fälle erklären, wo an Stelle des Auges bei gewissen Krebsen ein antennenähnliches Organ entsteht? Da diese Antenne denen gleich ist, die das Tier sonst besitzt, sollen wir nun vielleicht annehmen, daß es friber einmal Antennen an Stelle der Augen gehabt habe? " Weiter sagt MoszKowskr: "In einigen Fällen hat man bei der Regeneration des Salamanderbeines eine oder mehrere iuberzählige Zehen gefunden und auch dies ist als Ruickkehr zu einer ancestralen Form gedeutet worden. Nun hat aber ToRNren nachgewiesen, dab man nach Belieben überzählige Finger oder selbst eine ganze überzählige Hand erzeugen kann, indem man das Bein in ganz bestimmter Weise verwundet. *

Ich muß bemerken, daß ich in Moszkowskis Darlegung. keine genïgende Beweiskraft finde. Alle obigen und dergleichen Fälle, die als typische Heteromorphosen betrachtet werden miissen, entsprechen denjenigen Fällen in der normalen Ontogenese, welche als coenogenetische, also durch spezielle Entwicklungsbedingungen hervorgerufene Prozesse bezeichnet werden und nicht den phylogenetischen Stadien entsprechen.

Obwohl das biogenetische Grundgesetz als etwas nicht ad oculos beweisbares (mit gleichem Rechte müßten wir übrigens anch die ganze Abstammungslehre, die historische Geologie und die Weltgeschichte über den Haufen werfen) von manchen modernen Zoologen ganz abgelehnt wird, so beweisen jedoch solche Tatsachen, wie z. B. das Vorhandensein von gegabelten Extremitäten bei den Isopodenembryonen, die Existenz von Kiemenbogen, Kiementaschen, arteriellen Gefäßbogen, von Chorda dorsalis usw. bei den Embryonen höherer Vertebraten, das Vorhandensein des Schlüsselbeines bei Huftierembryonen, des Os centrale beim Menschenembryo, der provisorischen Zähne bei manchen Waltieren usw. usw., daß in der Ontogenie wirklich sehr viele ziellose morphologisehe Verhältnisse von hoher phylogenetischer Bedentung hervortreten. Wie es aber in der Ontogenie ganz verfehlt wäre, ïberall phylogenetische Momente zu erblicken, z. B. in dem Vorhandensein des Amnios, da auf Grund der vergleichenden Anatomie die palingenetischen von den coenogenetischen Prozessen kritisch unterschieden werden müssen, was 
jedoch nicht immer gelingt, so wäre es auch ganz unkritisch, allen heteromorphotischen Regenerationsprozessen einen gewissen phylogenetischen Sinn zuschreiben zu wollen.

Die regenerativen Prozesse verlaufen jedoch in vielen Fällen so ähnlich den embryonalen, daß wir mit großer Wahrscheinlichkeit annéhmen müssen, daß auch bei der Regeneration manche Erscheinungen von phylogenetischer Bedeutung hervortreten, was um so wahrscheinlicher ist, da beim Abtrennen ganzer Körperteile das Wundgewebe, befreit von benachbarten Einfliussen, in mancher Hinsicht in die Lage eines embryonalen Gewebes versetzt wird, so daß manche latente und bisher durch den Einfluß des ganzen Organismus unterdrückte Ánlagen leicht zur Äußerung gelangen können.

Als atavistische Erscheinungen von phylogenetischer Bedeutung. können wir jedoch nur diejenigen Regenerationsprozesse betrachten, welche 1) in gegebenem Falle bei verschiedensten Bedingungen der Regeneration mehr oder weniger gleich ausfallen, und 2) gewissen Verhältnissen bei phylogenetisch älteren Formen wirklich entsprechen. Diesen Bedingungen entspricht aber keineswegs z. B. die erwähnte, von Herbst beobachtete Heteromorphose bei manchen Crustaceen.

Die Art und Weise des Regenerationsganges ist von vielen inneren und äußeren Faktoren abhängig, die zum größten Teile noch unbekannt sind; es ist daher schwer verständlich, warum MongaN und Moszkowskr die in der Ontogenie so höchst bedeutenden phylogenetischen, auf den allgemeinen Vererbungsgesetzen beruhenden Faktoren bei den Regenerationsprozessen aprioristisch a limine abzulehnen suchen.

Ob die überzähligen Anhänge, die ich in meinem Falle in der linken Schere des Flußkrebses beschrieben habe, wirklich infolge eines Regenerationsprozesses entstanden sind, das ist meinerseits, wie ich nochmals ausdruicklich betone, nur eine wahrscheinliche Annahme. Die ïberzähligen Scheren könnten ja hier ganz analog. den regeneratorisch uberzähligen Fingern, den regeneratorisch uiberzähligen Schwänzen und überzähligen Köpfen zur Bildung gelangen. Entscheidend könnte hier übrigens nur ein Experiment sein. Interessant scheint mir jedoch die Tatsache zu sein, daß in meinem Falle die überzählige Schere ganz analoge morphologische Verhältnisse aufweist, wie die regenerierten kleineren Scheren und die normalen Scheren der phylogenetisch sehr wahrscheinlich älteren Formen der Flußkrebse. 